\title{
Theory of Local Times
}

\author{
Hitoshi Kitada \\ Department of Mathematical Sciences, University of Tokyo \\ Komaba, Meguro, Tokyo 153, Japan
}

(published in Il Nuovo Cimento - Vol. 109 B, N. 3 - March 1994 - pp. 281-302)

\begin{abstract}
Summary. - A model of a stationary universe is proposed. In this framework, time is defined as a local and quantum-mechanical notion in the sense that it is defined for each local and quantum-mechanical system consisting of finite number of particles. The total universe consisting of infinite number of particles has no time associated. It is a stationary bound state of the total Hamiltonian of infinite degrees of freedom. The quantum mechanics and the theory of general relativity are consistently united in this context if one uses this notion of local and quantum-mechanical time. As one of the consequences, the Einstein-Podolsky-Rosen paradox is resolved. The Hubble red-shift is explained as a consequence of general relativity which is consistent with quantum mechanics. This does not require us to argue on the beginning nor the end of the universe. The universe just exists without time.
\end{abstract}

PACS O3.65.Bz - Foundations, theory of measurement, miscellaneous theories.

\section{1. - Introduction.}

As stated in the abstract, the main theme of the paper is to present one possible consistent unification of quantum mechanics and general relativity. This is stated intentionally with anticipating the naive refutation that the Euclidean geometry which quantum mechanics follows and the non-flat Riemannian geometry which relativity follows can never be united consistently.

Our trick of the consistent unification of these two theories is to adopt a ten-dimensional vector bundle $X \times R^{6}$ (the reason $R^{6}$ is adopted instead of $R^{4}$ will be touched below) as the total physics space, where the base space $X$ and the fibre $R^{6}$ are mutually orthogonal. Quantum mechanics is set on the Euclidean space $R^{6}$ and relativity theory on the curved Riemannian space $X$. Each point $(t, x) \in X$ is correlated to the centre of mass of the local system consisting of finite number of (quantum-mechanical) particles, and these centres of mass are considered as the classical particles. These classical particles are regarded as moving following general relativity in the Riemannian manifold $X$ on the one hand, and the particles inside the local systems are regarded as moving following quantum mechanics on the other hand.

In this sense each point $(t, x)$ of the base Riemann space $X$ of the vector bundle $X \times R^{6}$ corresponds to the local system consisting of finite number of particles which follow quantum mechanics in each fibre $R^{6}$. 
Because the fibre $R^{6}$ where quantum mechanics holds and the base space $X$ where relativity holds are mutually orthogonal in the total space $X \times R^{6}$, it can be shown that the principles of quantum mechanics and the principles of general relativity are united consistently in this formulation, with the aid of the notion of quantum-mechanical local time introduced after sect. 2 below.

The reader may ask: There are many sublocal systems in a local system $(H, \mathcal{H})$, and the centres of mass of these sublocal systems follow classical relativity. But inside the local system $(H, \mathcal{H})$, quantum mechanics holds. Then what mechanics do these sublocal systems follow? The answer is

These sublocal systems follow classical relativity as far as the observer observes them in accordance with the observer's own time, but if the time of the system $(H, \mathcal{H})$ itself is adopted as the reference time, then the local system $(H, \mathcal{H})$ follows quantum mechanics.

Now here enters the notion of local time, which is the main ingredient of our consistent unification of the quantum and relativity theories.

Our starting point is the recognition that the time $t$ is given by the ratio $|x| /|v|$ of the position $x$ and the motion (velocity) $v$. This standpoint is fully explained in sect. 2 . This formulation is justified by the result (theorem l) of the many-particle scattering theory. Because of this definition of time, the Euclidean space of quantum mechanics becomes $R^{6}$ of $(x, v)$ instead of $R^{4}$ of $(t, x)$, and the usual four-dimensional structure $R^{4}$ is recovered as an approximation through the uncertainty principle stated in sect. 5 after definition 3 .

Like this, quantum mechanics controls the mechanics inside each local system, and the relative motion of the centres of mass of these local systems follows general relativity. In actual observations or experiments, the observer's time must be transformed into the local time of the observed local system. The rules of this transformation are given by the covariance principle and the equivalence principle of general relativity. An actual example of this kind of explanation of the relativistic quantum phenomena is given in sect. 9 .

As a consequence, the EPR paradox is explained without contradiction, and Hubble's red-shift is explained even though our model is constructed on the basis of a stationary universe. As another result, the dark matter is unnecessary in our theory to explain the stability of galaxies, clusters of galaxies, and so on.

\section{2. - What is time?}

This question seems to have a history as long as that of mankind itself. In the literature of physics, in the seventeenth century time was defined by Newton as a kind of absolute notion. In the first decade of the twentieth century, a reflection on the notion of time was given by Einstein in his theory of special relativity. Later, Einstein gave a more profound reflection on space-time in his theory of general relativity. Even though these reflections required us to make a reconstruction of our notion of space-time on a very deep level, time together with space is still considered to give a reference frame according to which one measures the physical quantities like positions, motions, velocities, and so on: Matter determines the space-time structure of the universe according to the theory of general relativity. Nonetheless, the space-time remains as a frame according to which the position of each matter is determined. 
What we propose in this paper is a different point of view on the notion of time based on the following simple but overlooked observation: time does not appear until it is measured by some equipments, clocks. We need tools to know the time. This situation is different from the perception of the positions and motions, which are recognized directly by our senses. Even when we use some tools like a rule to measure the length of a thing, what we actually do is to see which scales of the rule coincide with both sides of the thing. The fundamental recognition done here is the perception of this coincidence, which cannot be done without our senses. The positions and motions are recognized through our senses in this sense. On the contrary, if one reflects the measurement procedure of time by clocks, one notices that he or she compares several motions or movements of matters, and takes the quotient of position and velocity. In fact, the usual (analog) clock measures time by the motion of its hands. We look at the hands, and recognize that one second passes if the second hand $\langle\langle$ moves $\rangle\rangle$ one $\langle\langle$ scale $\rangle\rangle$. We do not measure time directly by our senses, but we know time by perceiving the positions and motions of the hands of clocks. In this sense time is neither a quantity nor a frame given a priori. What exists first are the positions and movements of the matters relative to our own position. The perception of the positions and motions indicates an introduction of the common parameter in each system of matters consisting of finite number of particles. This parameter is called time and it is a local notion by nature. This recognition is our starting point.

In the twentieth century several theories of the universe have been investigated. Most of these are related with the theory of (general) relativity. Many trials have been made to $\langle\langle$ quantize $\rangle\rangle$ the theory of relativity. But it seems that none of them can be said to have succeeded. (See Ashtekar's Introduction of Ashtekar and Stachel [1] and Streater's paper in Brown-Harré [2] for the history of many trials including the recent ones. See also Glimm-Jaffe [3] for the point of view that quantum field theory is successful to a certain extent.) One category of such theories is represented by the stationary theories of the universe. Another category consists of non-stationary ones. The typical theory of the latter category is the so-called 〈〈big-bang $\rangle\rangle$ theory. Tracing in this direction, Hawking introduced, for instance, imaginary time.

In this article we introduce one stationary model of universe. This enables us to define the notion of (local) times. According to our theory, there exists the total universe which has no time associated. It is a stationary bound state of the total Hamiltonian with infinite degrees of freedom. Our theory is quantized from the beginning in this context. Relative coordinates and momenta are postulated to exist according to our reflection of the measuring procedure of time stated above.

From these postulates we define time as a local (or $\langle\langle$ glocal $\rangle\rangle)$ and quantum-mechanical quantity. Our theory may also be called a quantization of time in this sense. We further show that our theory is consistent with the theory of general relativity. According to the so-called EPR paradox (see Redhead [4], for instance), quantum mechanics and the theory of relativity cannot be consistently united, because the former denies the local causality which is a consequence of the latter, as far as we suppose that quantum mechanics is complete in the sense described below in the argument of the EPR paradox in sect. 8 . In this sense the unification of these two theories is impossible on the same level or on the same $\langle\langle$ plane $\rangle\rangle$. In our unification of these theories, we $\langle\langle$ orthogonalize $\rangle\rangle$, so to speak, these two theories, or set these theories on mutually $\langle\langle$ orthogonal planes $\rangle$, with 
the introduction of local times. We do not try to quantize the theory of relativity itself, as it has been tried in many theories of quantum fields. We just leave the theory of general relativity as a classical theory. What we actually do is to show that our theory of local quantum-mechanical times is consistent with classical relativity.

Our theory is basically the non-relativistic quantum mechanics 〈〈orthogonalized $\rangle$ to general relativity. The actual way of this orthogonalization is shown in sect. 6. We take the standpoint that nature follows quantum mechanics intrinsically, and the relativistic effects only appear associated with observation. Namely, gravitation is nothing but an outlook; it is no intrinsic nature of the universe according to our theory. Note that this standpoint is different from the standpoint of quantum gravity being discussed recently, e.g., in Ashtekar and Stachel [1], where it is implicitly supposed that the quantum and gravitational aspects of the universe are possible to be discussed on the same $\langle\langle$ plane $\rangle\rangle$ or on the same level. Our theory differs from this traditional standpoint.

The point of our theory of orthogonalization of quantum mechanics and relativity theory is summarized as follows:

The observer's coordinate system $\left(t^{0}, x^{0}\right)$ of $R^{4}$ is independent of the coordinate system $\left(t^{1}, x^{1}\right)$ of the observed system, according to our definition of local times $t^{1}, t^{0}$ (sect. $5)$. Thus the observed system can follow quantum mechanics with respect to the coordinate system $\left(t^{1}, x^{1}\right)$, as well as it can follow classical relativity with respect to the coordinate system $\left(t^{0}, x^{0}\right)$. In this sense, any local system can follow classical relativity when observed, and at the same time, it can follow quantum mechanics inwardly or intrinsically.

This is our key of the consistent unification of quantum mechanics and general relativity.

Accordingly, any local system behaves in classical-mechanical way as well as in quantummechanical way, depending on the choice of coordinate systems. For the relation between these two looks of the behaviour of local systems, we make the following fundamental assumption:

The observer can see only the motions of centres of mass of local systems, and these motions are observed following classical relativity. The quantum effects inside the local systems are unobservable directly, but can be deduced from these classical observations of the sublocal systems.

The method of deduction of the internal quantum mechanics from the classical observations which we propose is discussed in sect. 9 as a procedure of observation.

The unification of quantum and relativity theories in the above form means that we abandon the relativistic quantum field theory. A positive reason which supports this abandonment is that there seems to be only a trivial model in the axiomatic quantum field theory in actual four-dimensional space-time (see Streater's paper in [2]). Owing to this abandonment of quantum field theory, we are free from such problems as renormalizations, divergence problems, and so on, which constitute the fundamental difficulties of quantum field theories. The classical fields are not quantized relativistically in our theory. They are left as classical notions. The quantum fields are auxiliary tools, which will be introduced to treat the creation and annihilation phenomena (see conjecture (2) below). The quantum fields are confined to the explanation of the non-relativistic quantum phenomena. 
Summarizing, the most important result of the paper is that there exists at least one consistent theory which unites the non-relativistic quantum mechanics and general relativity. (As an example of other trials in this direction, we refer to Prugovečki [5], where a geometro-stochastic approach is adopted for the problem of the unification of quantum theory and general relativity.) Even in our approach, we can give an explanation of the so-called relativistic quantum phenomena. These phenomena will be explained as the consequences of the observation activities: the actual experimental data are different from their true quantum-mechanical values because of the relativistic effects of observations, and the observer has to calculate the true values from his experimental data through some relativistic considerations. This point will be discussed in sect. 9 as regards observation. The necessity of considering high-energy particle physics is not strong in our context, since the universe does not begin via something like the big bang. However, some of the high-energy phenomena related with relativistic quantum mechanics can be explained in our context. Hopefully, a full explanation of these phenomena would be given in the future.

\section{3. - Global axiom.}

As a foundation of the theory of times, we adopt a model of stationary universe. Differently from the usual stationary theories where the time of the universe is assumed to exist a priori, our universe $\phi$, which is an element of a Hilbert space $\mathcal{U}$, has no time associated. It is assumed as a stationary bound state of a Hamiltonian $H$ of infinite degrees of freedom. In this sense our universe $\phi$ is quantized from the beginning:

Axiom 1. There exists a (separable) Hilbert space of (possible) universes

$$
\mathcal{U}=\{\phi\}=\bigoplus_{n=0}^{\infty}\left(\bigoplus_{\ell=0}^{\infty} \mathcal{H}^{n}\right) \quad(\mathcal{H}^{n}=\underbrace{\mathcal{H} \otimes \cdots \otimes \mathcal{H}}_{n \text { factors }})
$$

with $\mathcal{H}$ being a separable Hilbert space, a set of self-adjoint operators (observables) $\mathcal{O}=$ $\{A\}$ of the form $A \phi=\left(A_{n \ell} \phi_{n \ell}\right)$ for $\phi=\left(\phi_{n \ell}\right) \in \mathcal{U}$, and a self-adjoint operator $H \in \mathcal{O}$ in $\mathcal{U}$ such that for some $\phi \in \mathcal{U}-\{0\}$ and $\lambda \in R^{1}$

$$
H \phi=\lambda \phi
$$

in the following sense: There exists an infinite matrix $\left(\lambda_{n \ell}\right)$ of real numbers such that $H_{n \ell} \phi_{n \ell}=\lambda_{n \ell} \phi_{n \ell}$ for each $n \geq 1, \ell \geq 0$ and $\lambda_{n \ell_{n}} \rightarrow \lambda$ as $n \rightarrow \infty$ along any $\ell_{n}$ such that

$F_{n}^{\ell_{n}} \subset F_{n+1}^{\ell_{n+1}}$. Here $F_{n}$ is a finite subset of $\mathbf{N}=\{1,2, \cdots\}$ with $\sharp\left(F_{n}\right)=$ (the number of elements in $\left.F_{n}\right)=n$ and $\left\{F_{n}^{\ell}\right\}_{\ell=0}^{\infty}$ is the totality of such $F_{n}$.

$H$ is an infinite matrix $\left(H_{n \ell}\right)$ of self-adjoint operators $H_{n \ell}$ in $\mathcal{H}^{n}$. Axiom 1 asserts that this matrix converges in the sense of $(2)$.

We should repeat our standpoint here. Our purpose in this paper is to construct at least one consistent theory which unites quantum mechanics and relativity. As a mathematical problem, it is of course possible to consider the propagation $\exp [-i T H]$ along some global time $T$ in the grand universe $\mathcal{U}$. However, this does not seem to lead to any consistent unification of quantum mechanics and relativity. Axiom 1 is adopted so that it gives a starting point of our consistent unification. 


\section{4. - Local axioms.}

What we can perceive in the world are the (local) positions and movements of other matters relative to our own position. Comparing various movements, we determine time as a ratio of the positions and the movements (velocities). So there exist first the local and relative positions and velocities or momenta. This fact is formulated as in the following axiom.

Axiom 2. Let $n \geq 1$ and $F_{n+1}$ be a finite subset of $\mathbf{N}=\{1,2, \cdots\}$ with $\sharp\left(F_{n+1}\right)=$ $n+1$. Then for any $j \in F_{n+1}$, there exist self-adjoint operators $X_{j}=\left(X_{j 1}, X_{j 2}, X_{j 3}\right)$, $P_{j}=\left(P_{j 1}, P_{j 2}, P_{j 3}\right)$ in $\mathcal{H}^{n}$ and constants $m_{j}>0$ such that

$$
\begin{gathered}
{\left[X_{j \ell}, X_{k m}\right]=0, \quad\left[P_{j \ell}, P_{k m}\right]=0, \quad\left[X_{j \ell}, P_{k m}\right]=i \delta_{j k} \delta_{\ell m},} \\
\sum_{j \in F_{n+1}} m_{j} X_{j}=0, \quad \sum_{j \in F_{n+1}} P_{j}=0 .
\end{gathered}
$$

By the Stone-von Neumann theorem, axiom 2 also specifies the space dimension (see Abraham-Marsden [6], p.452). We identify $\mathcal{H}^{n}$ with $L^{2}\left(R^{3 n}\right)$ in the following.

What we want to mean by $H_{n \ell}(n, \ell \geq 0)$ in axiom 1 is the $N=(n+1)$-body Hamiltonian in the usual quantum mechanics. For the local Hamiltonian $H_{n \ell}$ we thus make the following postulate.

Axiom 3. Let $n \geq 0$ and $F_{N}(N=n+1)$ be a finite subset of $\mathbf{N}=\{1,2, \cdots\}$ with $\sharp\left(F_{N}\right)=N$. Let $\left\{F_{N}^{\ell}\right\}_{\ell=0}^{\infty}$ be the totality of such $F_{N}$. Then the Hamiltonians $H_{n \ell}(\ell \geq 0)$ are of the form

$$
H_{n \ell}=H_{n \ell 0}+V_{n \ell}, \quad V_{n \ell}=\sum_{\substack{\alpha=(i, j) \\ 1 \leq i<j<\infty, i, j \in F_{N}^{\ell}}} V_{\alpha}\left(x_{\alpha}\right)
$$

on $C_{0}^{\infty}\left(R^{3 n}\right)$, where $x_{\alpha}=x_{i}-x_{j}$ with $x_{i}$ being the position vector of the $i$-th particle, and $V_{\alpha}\left(x_{\alpha}\right)$ is a real-valued measurable function of $x_{\alpha} \in R^{3}$ which is $H_{n \ell 0}$-bounded with $H_{n \ell 0}$-bound of $V_{n \ell}$ less than 1. $H_{n \ell 0}=H_{(N-1) \ell 0}$ is the free Hamiltonian of the $N$-particle system. The concrete form is expressed as in (1.4) of ref. [7], if one uses clustered Jacobi coordinates.

This axiom implies that $H_{n \ell}=H_{(N-1) \ell}$ is uniquely extended to a self-adjoint operator in $\mathcal{H}^{n}=\mathcal{H}^{N-1}=L^{2}\left(R^{3(N-1)}\right)$ by the Kato-Rellich theorem.

\section{5. - Local times.}

For the $N$-body Hamiltonian $H_{N-1}=H_{n \ell}(N=n+1)$ the following theorem 1 is known [8] to hold under suitable assumptions on the pair potentials (assumption 1 in [9]) and on the decay property of subsystem eigenvectors (assumption 2 in [9]).

We here follow the notation and conventions in $[7,9]$ for the $N$-body quantum systems. In particular $H_{b}=H_{(N-1) b}=H_{N-1}-I_{b}=H_{n \ell}^{b}+T_{n \ell b}=H^{b}+T_{b}$ is the truncated 
Hamiltonian for the cluster decomposition $1 \leq|b| \leq N$, and $P_{b}^{M}$ is the $M$-dimensional partial projection of the eigenprojection $P_{b}=P_{H^{b}}$ associated with the subsystem $H^{b}$, i.e. $P_{b}$ is the orthogonal projection in $\mathcal{H}^{b}=L^{2}\left(R^{3(N-|b|)}\right)$ onto the eigenspace of $H^{b}$. $q_{b}$ is the velocity conjugate to the intercluster coordinates $x_{b}$. We define for a $k$-dimensional multi-index $M=\left(M_{1}, \cdots, M_{k}\right), M_{j} \geq 1$,

$$
\hat{P}_{k}^{M}=\left(I-\sum_{|b|=k} P_{b}^{M_{k}}\right) \cdots\left(I-\sum_{|d|=2} P_{d}^{M_{2}}\right)\left(I-P^{M_{1}}\right), \quad k=1, \cdots, N-1,
$$

where $P^{M_{1}}=P_{a_{1}}^{M_{1}}$ with $|a|=1$, and for a $|b|$-dimensional multi-index $M_{b}=\left(M_{1}, \cdots\right.$, $\left.M_{|b|-1}, M_{|b|}\right)=\left(\hat{M}_{b}, M_{|b|}\right)$

$$
\tilde{P}_{b}^{M_{b}}=P_{b}^{M_{|b|}} \hat{P}_{|b|-1}^{\hat{M}_{b}}, \quad 2 \leq|b| \leq N .
$$

It is clear that

$$
\sum_{2 \leq|b| \leq N} \tilde{P}_{b}^{M_{b}}=I-P^{M_{1}}
$$

provided that the component $M_{k}$ of $M_{b}$ depends only on the number $k$ but not on $b$. In the following we use such $M_{b}$ 's only. Under these circumstances, the following is known to hold.

Theorem 1 ([8]). Let assumptions 1 and 2 in [9] be satisfied. Let $f \in \mathcal{H}^{N-1}$. Then there is a sequence $t_{m} \rightarrow \pm \infty($ as $m \rightarrow \pm \infty)$ and a sequence $M_{b}^{m}$ of multi-indices whose components all tend to $\infty$ as $m \rightarrow \pm \infty$ such that for all cluster decompositions $b, 2 \leq$ $|b| \leq N, \psi \in C_{0}^{\infty}\left(R^{1}\right)$, and $\varphi \in C_{0}^{\infty}\left(R^{3(|b|-1)}\right)$,

$$
\begin{gathered}
\left\|\frac{\left|x^{b}\right|^{2}}{t_{m}^{2}} \tilde{P}_{b}^{M_{b}^{m}} \exp \left[-i t_{m} H_{N-1}\right] f\right\| \rightarrow 0, \\
\left\|\left\{\psi\left(H_{N-1}\right)-\psi\left(H_{b}\right)\right\} \tilde{P}_{b}^{M_{b}^{m}} \exp \left[-i t_{m} H_{N-1}\right] f\right\| \rightarrow 0, \\
\left\|\left\{\varphi\left(x_{b} / t_{m}\right)-\varphi\left(q_{b}\right)\right\} \tilde{P}_{b}^{M_{b}^{m}} \exp \left[-i t_{m} H_{N-1}\right] f\right\| \rightarrow 0
\end{gathered}
$$

as $m \rightarrow \pm \infty$.

Definition 1. Let $\phi=\left(\phi_{n \ell}\right)$ with $\phi_{n \ell}=\phi_{n \ell}\left(x_{1}, \cdots, x_{n}\right) \in L^{2}\left(R^{3 n}\right)$ be the universe in axiom 1. We define $\mathcal{H}_{n \ell}$ as the sub-Hilbert space of $\mathcal{H}^{n}$ generated by the functions $\phi_{m k}\left(x^{(\ell)}, y\right)$ of $x^{(\ell)} \in R^{3 n}$, by regarding $y \in R^{3(m-n)}$ as a parameter, where $m \geq n$, $F_{n+1}^{\ell} \subset F_{m+1}^{k}$, and $x^{(\ell)}$ are the (relative) coordinates of $(n+1)$ particles in $F_{n+1}^{\ell}$. $\mathcal{H}_{n \ell}$ is called a local universe of $\phi . \mathcal{H}_{n \ell}$ is said to be non-trivial if $\left(I-P_{H_{n \ell}}\right) \mathcal{H}_{n \ell} \neq\{0\}$.

The total universe $\phi$ is a single element in $\mathcal{U}$. The local universe $\mathcal{H}_{n \ell}$ may be richer. This is because we consider the subsystems of the universe consisting of a finite number 
of particles. These subsystems receive the influence from the other particles of infinite number outside the subsystems, and may vary to constitute a non-trivial subspace $\mathcal{H}_{n \ell}$.

Definition 2. The restriction of $H$ to $\mathcal{H}_{n \ell}$ is also denoted by the same notation $H_{n \ell}$ as the $(n, \ell)$-th component of $H$. We call the pair $\left(H_{n \ell}, \mathcal{H}_{n \ell}\right)$ a local system. The unitary group $\exp \left[-i t H_{n \ell}\right]\left(t \in R^{1}\right)$ on $\mathcal{H}_{n \ell}$ is called the proper clock of the local system $\left(H_{n \ell}, \mathcal{H}_{n \ell}\right)$, if $\mathcal{H}_{n \ell}$ is non-trivial: $\left(I-P_{H_{n \ell}}\right) \mathcal{H}_{n \ell} \neq\{0\}$. (Note that the clock is defined only for $N=n+1 \geq 2$, since $H_{0 \ell}=0$.) The universe $\phi$ is called rich if $\mathcal{H}_{n \ell}$ equals $\mathcal{H}^{n}=L^{2}\left(R^{3 n}\right)$ for all $n \geq 1, \ell \geq 0$. For a rich universe $\phi, H_{n \ell}$ equals the $(n, \ell)$-th component of $H$.

The formula (11) indicates that $t_{m}$ is asymptotically equal to $\pm\left|x_{b}\right| /\left|q_{b}\right|$ as $m \rightarrow \pm \infty$, independently of the choice of cluster decompositions $b$. This is precisely the actual procedure of measuring the time $t_{m}$ in mechanics. The implication of this theorem is therefore interpreted as follows: If one $\langle\langle$ measures $\rangle\rangle$ the time of a state $f \in(I-$ $\left.P_{H_{(N-1) \ell}}\right) \mathcal{H}_{(N-1) \ell}-\{0\}$ in the local system $\left(H_{(N-1) \ell}, \mathcal{H}_{(N-1) \ell}\right)$ by the associated proper clock $\exp \left[-i t H_{(N-1) \ell}\right] f$, namely if one measures the quotient $\pm\left|x_{b}\right| /\left|q_{b}\right|$ of the scattered particles which are regarded as moving almost in a steady velocity, then that time is asymptotically equal to the parameter $t_{m}$ in the exponent of $\exp \left[-i t_{m} H_{(N-1) \ell}\right] f$ as $m \rightarrow \pm \infty$. In this sense $t_{m}$ is interpreted as the quantum-mechanical proper time of the local system $\left(H_{n \ell}, \mathcal{H}_{n \ell}\right)=\left(H_{(N-1) \ell}, \mathcal{H}_{(N-1) \ell}\right)$, if $\left(I-P_{H_{(N-1) \ell}}\right) \mathcal{H}_{(N-1) \ell} \neq\{0\}$.

Definition 3. The parameter $t$ in the exponent of the proper clock $\exp \left[-i t H_{n \ell}\right]=$ $\exp \left[-i t H_{(N-1) \ell}\right]$ of the local system $\left(H_{n \ell}, \mathcal{H}_{n \ell}\right)$ is called the (quantum-mechanical) proper time or local time of the local system $\left(H_{n \ell}, \mathcal{H}_{n \ell}\right)$, if $\left(I-P_{H_{n \ell}}\right) \mathcal{H}_{n \ell} \neq\{0\}$. This time $t$ is denoted by $t_{\left(H_{n \ell}, \mathcal{H}_{n \ell}\right)}$ indicating the local system under consideration.

This definition is the reverse to the usual definition of the motion or dynamics of the $N$-body quantum systems, where the time $t$ is given a priori and the motion of the particles is defined by $\exp \left[-i t H_{(N-1) \ell}\right] f$ for a given initial state $f$ of the system.

We notice here that there are two possible 〈〈directions $\rangle$ or $\langle\langle$ orientations $\rangle\rangle$ of time $t$; the one where $t$ increases to $+\infty$, and the other where $t$ decreases to $-\infty$. So far discussed, one can choose an arbitrary orientation from them depending on each local system. However, the axiom 4 (the general principle of relativity) which will be introduced below determines the orientation of time to be common to all local systems (see HawkingEllis [10], p.181).

Time is thus defined only for the local systems $\left(H_{n \ell}, \mathcal{H}_{n \ell}\right)$ and is determined by the associated proper clock $\exp \left[-i t H_{n \ell}\right]$. Therefore, there are infinitely many times $t=$ $t_{\left(H_{n \ell}, \mathcal{H}_{n \ell}\right)}$ each of which is proper to the local system $\left(H_{n \ell}, \mathcal{H}_{n \ell}\right)$. In this sense time is a local notion. There is no time for the total universe $\phi$ in axiom 1 , which is a (stationary) bound state for the total Hamiltonian $H$.

This local time is an approximate one in a double sense: First, $t_{m}$ is only asymptotically equal to $\pm\left|x_{b}\right| /\left|q_{b}\right|$ as $m \rightarrow \pm \infty$. This fact explains the so-called principle of uncertainty in our context. In the usual explanation, the position $x_{b}$ and the velocity $q_{b}$ or the momentum $p_{b}$ cannot be determined with equal accuracy. According to our theory, this is rephrased as follows: The time $t$ cannot be determined accurately, even if $x_{b}$ and $q_{b}$ could be determined precisely. It is only determined in some mean sense as in (11). (See Enss 
[8]. Also see Dereziński [11], sect. 5, for more precise inequalities which hold for $x_{b}, q_{b}$ and the local time $t$ under some decompositions of the phase space other than (8).) Second, the local Hamiltonian $H_{n \ell}$ is not the total Hamiltonian $H$. Or rather, the time arises from this approximation of $H$ by $H_{n \ell}$. This approximation may make $\mathcal{H}_{n \ell}$ non-trivial, and the clock $\exp \left[-i t H_{n \ell}\right]$ can be defined as in definition 2 owing to $\left(I-P_{H_{n \ell}}\right) \mathcal{H}_{n \ell} \neq\{0\}$. On the contrary, the total universe $\phi$ has no associated clock and time, since $\left(I-P_{H}\right) \phi=0$.

Our theory of local times further implies in particular that local systems $\left(H_{n \ell}, \mathcal{H}_{n \ell}\right)$ cannot be decomposed into pieces and are mutually independent: It is true that for a subset $F_{N^{\prime}}^{\ell^{\prime}} \subset F_{N}^{\ell}$ with $N^{\prime}<N, H_{\left(N^{\prime}-1\right) \ell^{\prime}}$ is a subsystem Hamiltonian of $H_{(N-1) \ell}$. However, the corresponding times $t_{N^{\prime} \ell^{\prime}}$ and $t_{N \ell}$ are measured mutually independently as in theorem 1 . Namely, the clocks $\exp \left[-i t H_{\left(N^{\prime}-1\right) \ell^{\prime}}\right]$ and $\exp \left[-i t H_{(N-1) \ell}\right]$ are different. More precisely speaking, the base Hilbert spaces $\mathcal{H}_{\left(N^{\prime}-1\right) \ell^{\prime}}$ and $\mathcal{H}_{(N-1) \ell}$ have different representations $L^{2}\left(R^{3\left(N^{\prime}-1\right)} ; N^{\prime}, \ell^{\prime}\right)$ and $L^{2}\left(R^{3(N-1)} ; N, \ell\right)$ in general even on the common configuration space $R_{x\left(\ell^{\prime}\right)}^{3\left(N^{\prime}-1\right)}$. Thus the corresponding $\left|x_{b}^{\prime}\right| /\left|q_{b}^{\prime}\right|$ and $\left|x_{b}\right| /\left|q_{b}\right|$ are not correlated in general. The same is true for two arbitrary different local systems $\left(H_{n \ell}, \mathcal{H}_{n \ell}\right)$ and $\left(H_{m k}, \mathcal{H}_{m k}\right)$.

The present theory differs from most of the existing theories of the universe in this point. They start from the existence of particles or material points obtained by dividing matters into pieces infinitesimally. In our theory the local systems are regarded as the generic points or as the classical particles and cannot be divided further, because any divisions vary the associated space-time correspondingly. In this sense each local system $\left(H_{n \ell}, \mathcal{H}_{n \ell}\right)$ is a $\langle\langle$ glocal $\rangle\rangle$ existence: It is neither a local thing nor a global one.

We have defined the (local) time $t=t_{\left(H_{n \ell}, \mathcal{H}_{n \ell}\right)}$ for each local system $\left(H_{n \ell}, \mathcal{H}_{n \ell}\right)$. This time $t$ satisfies theorem 1-(11). If one regards the time $t$ as a given quantity, this fact is interpreted as follows: In each local system $\left(H_{n \ell}, \mathcal{H}_{n \ell}\right)$, physics follows quantum mechanics, i.e. it follows the Schrödinger equation.

Our definition of times is consistent with the theory of (general) relativity of Einstein. Our (quantum-mechanical) proper time of the local system $\left(H_{n \ell}, \mathcal{H}_{n \ell}\right)$ can be regarded as the quantum-mechanical correspondent to the classical proper time in the theory of relativity. Within the local system $\left(H_{n \ell}, \mathcal{H}_{n \ell}\right)$ the velocity $q_{b}$ can be arbitrarily large. This is because one uses the proper time of the system $\left(H_{n \ell}, \mathcal{H}_{n \ell}\right)$. If one measures the velocity of other systems from one's own system using the associated proper time, then those other systems move in accordance with the relativity theory.

\section{6. - Relativity.}

For the relative motions of the centres of mass of local systems, we postulate the principle of (general) relativity and the principle of equivalence as in Einstein [12].

What should be stated first on our introduction of relativity is that only the relative classical motions of the centres of mass of local systems are observable in our theory. The internal quantum-mechanical motion within each local system is independent of observation, at least at the present stage of our theory till sect. 9. In this sense, the internal quantum-mechanical motion within a local system is unobservable. We postulate axiom 6 in sect. 9, which gives a principle of the deduction of the internal quantum-mechanical motion within each local system from classical observations of its sublocal systems, through certain relativistic considerations. 
Following definition 3 and eqs.(4) of axiom 2, we define the local space-time $(t, x)=$ $\left(t_{\left(H_{n \ell}, \mathcal{H}_{n \ell}\right)}, x_{\left(H_{n \ell}, \mathcal{H}_{n \ell}\right)}\right)$ with $x=\left(x_{1}, x_{2}, x_{3}\right) \in R^{3}$ of the local system $\left(H_{n \ell}, \mathcal{H}_{n \ell}\right)$ such that the centre of mass of the local system $\left(H_{n \ell}, \mathcal{H}_{n \ell}\right)$ is at the origin of the space coordinates $x=\left(x_{1}, x_{2}, x_{3}\right) \in R^{3}$. In this sense time $t=t_{\left(H_{n \ell}, \mathcal{H}_{n \ell}\right)}$ is interpreted as the relativistic proper time associated with the centre of mass of the system $\left(H_{n \ell}, \mathcal{H}_{n \ell}\right)$. Once the local space-time $(t, x)=\left(t_{\left(H_{n \ell}, \mathcal{H}_{n \ell}\right)}, x_{\left(H_{n \ell}, \mathcal{H}_{n \ell}\right)}\right)$ has been defined, one can $o b$ serve the movements of other systems from this space-time coordinates. The space-time $\left(t_{\left(H_{m k}, \mathcal{H}_{m k}\right)}, x_{\left(H_{m k}, \mathcal{H}_{m k}\right)}\right)$ of the other system $\left(H_{m k}, \mathcal{H}_{m k}\right)$ is defined independently of that of system $\left(H_{n \ell}, \mathcal{H}_{n \ell}\right)$. There is no quantum-mechanical correlation between two localcoordinate systems $\left(t_{\left(H_{n \ell}, \mathcal{H}_{n \ell}\right)}, x_{\left(H_{n \ell}, \mathcal{H}_{n \ell}\right)}\right)$ and $\left(t_{\left(H_{m k}, \mathcal{H}_{m k}\right)}, x_{\left(H_{m k}, \mathcal{H}_{m k}\right)}\right)$ unless one, or the observer, unites those two systems $\left(H_{n \ell}, \mathcal{H}_{n \ell}\right)$ and $\left(H_{m k}, \mathcal{H}_{m k}\right)$ in a single system for his particular purposes of observation. (We remark that the combined or united system exists a priori by definition 2, independently of the observer's concern.) In this case, the coordinates of the resulting combined system $\left(H_{p j}, \mathcal{H}_{p j}\right)$ is again independent of those of the subsystems $\left(H_{n \ell}, \mathcal{H}_{n \ell}\right)$ and $\left(H_{m k}, \mathcal{H}_{m k}\right)$. Thus the quantum mechanics which governs the inside of each local system puts no restriction on the relative motions of the centres of mass of local systems, as will be seen in theorem 2 below. Therefore, there is no reason to exclude classical mechanics in describing the relative motions of the centres of mass of other systems observed in one's coordinate system $(t, x)=\left(t_{\left(H_{n \ell}, \mathcal{H}_{n \ell}\right)}, x_{\left(H_{n \ell}, \mathcal{H}_{n \ell}\right)}\right)$.

Axiom 4. Those laws of physics which control the relative motions of the centres of mass of the observed local systems are expressed by the classical equations which are covariant under the change of observer's coordinate systems of $R^{4}:(t, x)=\left(t_{\left(H_{m k}, \mathcal{H}_{m k}\right)}, x_{\left(H_{m k}, \mathcal{H}_{m k}\right)}\right)$ to $(t, x)=\left(t_{\left(H_{n \ell}, \mathcal{H}_{n \ell}\right)}, x_{\left(H_{n \ell}, \mathcal{H}_{n \ell}\right)}\right)$ for any pairs $(m, k),(n, \ell)$.

It is included in this axiom that one can observe the positions of other systems (i.e. their centres of mass) in his coordinate system $(t, x)$. The relative velocities of the observed systems are then defined as quotients of the relative positions of those systems and the (local and quantum-mechanical) time $t$ of one's own or the observer's system. These are our definitions of the measurement procedure of classical quantities, which accord with the ordinary (implicit) agreement among physicists where the time is given a priori. (This is the point where we reversed the order of the time and the velocity in our definitions of quantum-mechanical times.)

Axiom 5 . The coordinate system $\left(t_{\left(H_{n \ell}, \mathcal{H}_{n \ell}\right)}, x_{\left(H_{n \ell}, \mathcal{H}_{n \ell}\right)}\right)$ associated with the local system $\left(H_{n \ell}, \mathcal{H}_{n \ell}\right)$ is the local Lorentz system of coordinates. Namely, the gravitational potentials $g_{\mu \nu}$ for the centre of mass of the local system $\left(H_{n \ell}, \mathcal{H}_{n \ell}\right)$, observed in these coordinates $\left(t_{\left(H_{n \ell}, \mathcal{H}_{n \ell}\right)}, x_{\left(H_{n \ell}, \mathcal{H}_{n \ell}\right)}\right)$, are equal to $\eta_{\mu \nu}$. Here $\eta_{\mu \nu}=0(\mu \neq \nu),=1(\mu=\nu=1,2,3)$, and $=-1(\mu=\nu=0)$.

For the field equation which determines the metric $g_{\mu \nu}$, we refer to Hawking-Ellis [10], p.74 or Friedman [3], p.180.

Axiom 5 implies that for the coordinate system $\left(t_{\left(H_{n \ell}, \mathcal{H}_{n \ell}\right)}, x_{\left(H_{n \ell}, \mathcal{H}_{n \ell}\right)}\right)$ associated with the local system $\left(H_{n \ell}, \mathcal{H}_{n \ell}\right)$, the principle of constancy of light velocity holds in the following special sense: The light radiated from another system $\left(H_{m k}, \mathcal{H}_{m k}\right)$ moving with a steady velocity relative to one's own system $\left(H_{n \ell}, \mathcal{H}_{n \ell}\right)$ propagates through the flat region where $g_{\mu \nu}=\eta_{\mu \nu}$, at a constant speed independent of the velocity of the system $\left(H_{m k}, \mathcal{H}_{m k}\right)$ relative to one's own system $\left(H_{n \ell}, \mathcal{H}_{n \ell}\right)$. 


\section{Theorem 2. Axioms 4 and 5 are consistent with axioms 1-3.}

Proof. This may be clear by the mutual independence of local systems and the associated times stated at the end of the previous section: We can assume any equations on relative motions, which follow arbitrarily given transformation rules under the change of the coordinates systems between any two local systems, and give arbitrary numbers to $g_{\mu \nu}$, as far as these are consistent with $\langle\langle$ physics $\rangle\rangle$. Whichever equations may one adopt to govern the relative motions of centres of mass of local systems, these equations are consistent with the quantum mechanics that controls the inside of the local systems.

For the sake of clarity, however, we repeat the argument of the previous section. The local coordinate system $\left(t_{\left(H_{n \ell}, \mathcal{H}_{n \ell}\right)}, x_{\left(H_{n \ell}, \mathcal{H}_{n \ell}\right)}\right)$ is determined only within each local system $\left(H_{n \ell}, \mathcal{H}_{n \ell}\right)$, through the quantum-mechanical internal motions of the system. This coordinate system is independent of the local coordinate system $\left(t_{\left(H_{m k}, \mathcal{H}_{m k}\right)}, x_{\left(H_{m k}, \mathcal{H}_{m k}\right)}\right)$ of any other local system $\left(H_{m k}, \mathcal{H}_{m k}\right)$. This is due to the mutual independence of the $L^{2}$ representations of the base Hilbert spaces $\mathcal{H}_{n \ell}$ and $\mathcal{H}_{m k}$.

The relativity axioms, axioms 4 and 5, are concerned merely with the centres of mass of local systems $\left(H_{m k}, \mathcal{H}_{m k}\right)$, observed by an observer system $\left(H_{n \ell}, \mathcal{H}_{n \ell}\right)$ with coordinate system $\left(t_{\left(H_{n \ell}, \mathcal{H}_{n \ell}\right)}, x_{\left(H_{n \ell}, \mathcal{H}_{n \ell}\right)}\right)$. This observer's coordinate system $\left(t_{\left(H_{n \ell}, \mathcal{H}_{n \ell}\right)}, x_{\left(H_{n \ell}, \mathcal{H}_{n \ell}\right)}\right)$ is independent of the coordinate system $\left(t_{\left(H_{m k}, \mathcal{H}_{m k}\right)}, x_{\left(H_{m k}, \mathcal{H}_{m k}\right)}\right)$ of the observed system $\left(H_{m k}, \mathcal{H}_{m k}\right)$, as stated in the previous paragraph. Because of this independence, the system $\left(H_{m k}, \mathcal{H}_{m k}\right)$ can follow quantum mechanics (axioms 1-3) inside the system with respect to its own coordinate system $\left(t_{\left(H_{m k}, \mathcal{H}_{m k}\right)}, x_{\left(H_{m k}, \mathcal{H}_{m k}\right)}\right)$, as well as its centre of mass can follow general relativity (axiom 4) or any other given postulates with respect to the observer's coordinate system $\left(t_{\left(H_{n \ell}, \mathcal{H}_{n \ell}\right)}, x_{\left(H_{n \ell}, \mathcal{H}_{n \ell}\right)}\right)$. This is the case, even if the coordinate system $\left(t_{\left(H_{n \ell}, \mathcal{H}_{n \ell}\right)}, x_{\left(H_{n \ell}, \mathcal{H}_{n \ell}\right)}\right)$ of the observer coincides with the coordinate system $\left(t_{\left(H_{m k}, \mathcal{H}_{m k}\right)}, x_{\left(H_{m k}, \mathcal{H}_{m k}\right)}\right)$ of the observed system itself, because the motion of the centre of mass and the internal relative motion of a local system are mutually independent. Therefore, the local Lorentz postulate (axiom 5) of the centre of mass of the system $\left(H_{n \ell}, \mathcal{H}_{n \ell}\right)$ also does not contradict the Euclidean postulates in sect. 3-5 of the internal space-time of that system.

In this sense, axioms 4 and 5 are chosen so that the relativity theory holds between the observed motions of centres of mass of local systems, and have nothing to do with the internal motion of each local system, which obeys axioms 1-3. Thus axioms 4 and 5 are consistent with axioms 1-3.

Remark. As stated in the introduction, the theory of local times can be reformulated or redescribed as a kind of vector bundle theory with ten-dimensional total space $X \times R^{6}$ and four-dimensional base space $X . R^{6}$ corresponds to the Euclidean space of quantum mechanics inside the local systems. $X$ is the Riemannian manifold with metric $g_{\mu \nu}$, the gravitational potentials. $X$ corresponds to the classical space-time observed by a fixed observer, which gives the observer's reference frame or coordinate system for measurements of the classical particles, the centres of mass of other local systems. Theorem 1 due to Enss is interpreted as a contraction procedure of the fibre $R^{6}$ to four-dimensional space-time $R^{4}$ in an approximate context of the uncertainty principle described in sect. 5 after definition 3. Owing to the orthogonality of $X$ and $R^{6}$ in the total space $X \times R^{6}$, the quantum and relativity theories hold in $R^{6}$ and in $X$, respectively, without mutual 
contradiction.

This is the mathematical explanation of the consistency of axioms 1-5, namely the proof of theorem 2 .

This proof describes the way of the 〈〈orthogonalization〉〉 of quantum mechanics and relativity, as announced in sect. 2 .

Under axiom 5 , the local time $t_{\left(H_{n \ell}, \mathcal{H}_{n \ell}\right)}$ of the system $\left(H_{n \ell}, \mathcal{H}_{n \ell}\right)$ coincides with the relativistic proper time of the centre of mass of the system, because, at the centre of mass, the space coordinates $x_{\left(H_{n \ell}, \mathcal{H}_{n \ell}\right)}=0$.

The relation of the internal motion of a local system with the motion of centres of mass of its subsystems will be discussed in sect. 9 which deals with observation.

Summing up, we have obtained the following physical picture of the universe: The universe $\phi$ is a stationary bound state of the total Hamiltonian $H$ with infinite degrees of freedom. Times $t=t_{\left(H_{n \ell}, \mathcal{H}_{n \ell}\right)}$ appear only for local systems $\left(H_{n \ell}, \mathcal{H}_{n \ell}\right)$. According to this time $t$, the physics laws within the local system $\left(H_{n \ell}, \mathcal{H}_{n \ell}\right)$ obey the quantum mechanics, and the physics laws outside the system which govern the relative motions between the centres of mass of local systems obey the classical theory of relativity. These two sorts of view are consistent, because the quantum mechanics inside the local systems puts no restriction on the motions of the centres of mass of local systems owing to the mutual independence of local systems.

\section{7. - A paradox of cyclotron.}

As an illustration of the unification of quantum mechanics and relativity in our context, we consider the experiment by a cyclotron. To clarify the point of our argument, we consider an ideal situation.

We suppose that some electrons for example are accelerated by a cyclotron, and can have velocities very near the velocity of light. By this experiment the observer can $\langle\langle$ see $\rangle\rangle$ many phenomena: the electrons are accelerated to have the velocities near the velocity of light, hence some relativistic phenomena occur, and some of the electrons may hit a nucleus and they together produce or change into several elementary particles. From these observations, the observer $\langle\langle$ knows $\rangle\rangle$ the masses, velocities, energies, and so on, of these particles by some reasoning or analysis of the experimental data. In this experiment, the quantum-mechanical and relativistic effects look like appearing at the same $\langle\langle$ time $\rangle\rangle$. This might be taken as a contradiction: As we shall see in the next section, quantum mechanics yields non-locality, which contradicts the local causality deduced from relativity.

The answer to this problem is as follows: The local causality is only concerned with the observed relative motions between the centres of mass of local systems, e.g., between the system $\left(H_{n \ell}, \mathcal{H}_{n \ell}\right)$ of the electrons under the acceleration, and the system $\left(H_{m k}, \mathcal{H}_{m k}\right)$ of the stationary nucleus. In this case, the observed motion between the two systems naturally follows the relativity theory, and the causality holds, according to our theory. When the observer $\langle\langle$ watches $\rangle$ the collision of the accelerated electrons and the stationary nucleus, it looks at the inside of the combined local system $\left(H_{p j}, \mathcal{H}_{p j}\right)$ of the two systems $\left(H_{n \ell}, \mathcal{H}_{n \ell}\right)$ and $\left(H_{m k}, \mathcal{H}_{m k}\right)$. Since what can be observed is only the relative classical motions of the centres of mass of sublocal systems of $\left(H_{p j}, \mathcal{H}_{p j}\right)$, the observer cannot see the inside of the system $\left(H_{p j}, \mathcal{H}_{p j}\right)$. However, as we shall discuss in sect. 9 , one can deduce 
the internal quantum-mechanical motion within $\left(H_{p j}, \mathcal{H}_{p j}\right)$ from these observations of sublocal systems. This deduction leads one to conclude that the physics within the system $\left(H_{p j}, \mathcal{H}_{p j}\right)$ follows quantum mechanics, and he sees the quantum-mechanical phenomena of collision in $\left(H_{p j}, \mathcal{H}_{p j}\right)$.

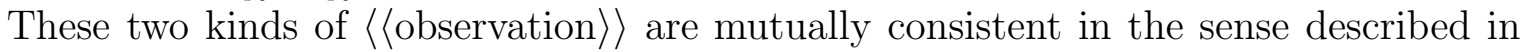
the proof of theorem 2. The observed motion between the centres of mass of the two systems $\left(H_{n \ell}, \mathcal{H}_{n \ell}\right)$ and $\left(H_{m k}, \mathcal{H}_{m k}\right)$ can follow classical relativity and causality, without any contradiction with the non-locality of quantum mechanics within the combined system $\left(H_{p j}, \mathcal{H}_{p j}\right)$. This consistency which follows from the mutual independence of the coordinate systems of the observer and the observed local systems is our solution of the unification of quantum mechanics and relativity. We shall analyse the problem in more detail in the following sections.

\section{8. - EPR paradox.}

Einstein-Podolsky-Rosen [14] argued that quantum mechanics (QM) combined with the locality principle (Redhead [4], p.75):

L) Elements of reality pertaining to one system cannot be affected by measurements performed $\langle\langle$ at a distance $\rangle$ on another system,

implies the incompleteness of quantum mechanics. Here completeness means that ([14])

C) Every element of the physical reality must have a counterpart in the physical theory.

This argument is rephrased as follows:

$$
\mathrm{QM} \Rightarrow \neg(\mathrm{L}) \quad \text { or } \quad \neg(\mathrm{C})
$$

which is called Einstein Dilemma in Redhead [4]. Here QM is the theory of quantum mechanics, and $\langle\langle\neg\rangle\rangle$ denotes the negation. We refer to Jammer [15], Selleri [16], Schommers [17] for further references.

Contrary to Einstein-Podolsky-Rosen [14], we adopt the standpoint that quantum mechanics is complete in the sense of $\mathrm{C}$ ). Thus quantum mechanics yields that non-locality holds within each local system. (There are arguments that the negation of the Bell inequality implies non-locality. Thus, in these arguments, quantum mechanics automatically yields non-locality. See d'Espagnat's paper in Schommers [17]. However, there are refutations to this type of arguments. See Redhead [4], chapt. 4 for instance.) In this sense, quantum mechanics contradicts local causality in the relativity theory (see the arguments in Redhead [4], p.75, also see Hawking-Ellis [10], chapt. 3). However, this situation is not a contradiction in our context of local times. The reason is that, in our theory, the classical theory of relativity is concerned only with the relative motions between the centres of mass of local systems, observed in the observer's coordinate system $(t, x)$. Thus, local causality is required only to the observed relative motions of the centres of mass of local systems, not to the physics within the local systems, which is unobservable directly according to our fundamental assumption made in sect. 2. This allows us to 
admit the unobservable non-locality within each local system, where quantum mechanics holds consistently with non-locality (by (12)).

For illustration, let us take the well-known example of two photons polarized mutually orthogonally. (See Redhead [4], chapt. 3 and sect. 4.5.) Let $\left(H_{n \ell}, \mathcal{H}_{n \ell}\right)$ and $\left(H_{m k}, \mathcal{H}_{m k}\right)$ be the local systems of each of these two photons, and let $\left(H_{p j}, \mathcal{H}_{p j}\right)$ be the combined system of the two photons. According to our theory, within this local system $\left(H_{p j}, \mathcal{H}_{p j}\right)$ of two photons, the interactions propagate with infinite speed by the quantum mechanics valid inside the system, as far as the two photons are considered to constitute one local system $\left(H_{p j}, \mathcal{H}_{p j}\right)$, and are $\langle\langle$ observed $\rangle\rangle$ from their creation to the measurements of their polarization traced along the proper local time $t_{\left(H_{p j}, \mathcal{H}_{p j}\right)}$. In this sense, non-locality is no contradiction within the combined local system of the two photons. Only when the two photons are considered as two different local systems $\left(H_{n \ell}, \mathcal{H}_{n \ell}\right)$ and $\left(H_{m k}, \mathcal{H}_{m k}\right)$ and are observed in the observer's coordinate system, the classical-mechanical speed of interactions appears and the situation looks like a contradiction. However, since $\left(H_{p j}, \mathcal{H}_{p j}\right)$, $\left(H_{n \ell}, \mathcal{H}_{n \ell}\right)$, and $\left(H_{m k}, \mathcal{H}_{m k}\right)$ are mutually independent quantum-mechanically in their respective local times, this situation is not a contradiction: The postulate that the relative motions between the centres of mass of $\left(H_{n \ell}, \mathcal{H}_{n \ell}\right)$ and $\left(H_{m k}, \mathcal{H}_{m k}\right)$ observed in the observer's coordinates is governed by classical relativity, hence the local causality holds between the two systems, does not contradict, by the mutual independence of the coordinates of these two systems, the fact that the combined system $\left(H_{p j}, \mathcal{H}_{p j}\right)$ follows quantum mechanics in its own local time $t_{\left(H_{p j}, \mathcal{H}_{p j}\right)}$, according to which non-locality holds.

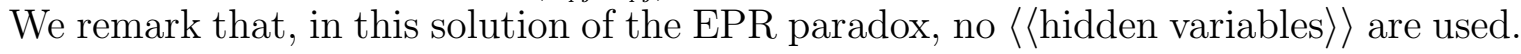

\section{9. - Observation.}

So far we have postulated five axioms, which describe the fundamental properties of the universe, and we have seen that these axioms are mutually consistent. We turn to the considerations of the observation process by an observer's system.

When the observer sees the universe, the potentials $V_{\alpha}\left(x_{\alpha}\right)$ of the observed systems effective between these local systems do not give any quantum-mechanical affection but should be interpreted as the classical potentials: A local system $\left(H_{n \ell}, \mathcal{H}_{n \ell}\right)$ is closed when it is considered within its own proper coordinate system $\left(t_{\left(H_{n \ell}, \mathcal{H}_{n \ell}\right)}, x_{\left(H_{n \ell}, \mathcal{H}_{n \ell}\right)}\right)$, since the time $t_{\left(H_{n \ell}, \mathcal{H}_{n \ell}\right)}$ is determined only within the system by the associated clock $\exp \left[-i t H_{n \ell}\right]$. Other local systems $\left(H_{m k}, \mathcal{H}_{m k}\right)$ can influence the local system $\left(H_{n \ell}, \mathcal{H}_{n \ell}\right)$ in the quantum-mechanical way only when these are considered by an observer as a single combined system of $\left(H_{n \ell}, \mathcal{H}_{n \ell}\right)$ and $\left(H_{m k}, \mathcal{H}_{m k}\right)$. Only at this stage the time is common to these two systems, and the quantum-mechanical correlation between these systems through pair potentials $V_{\alpha}\left(x_{\alpha}\right)$ can be discussed on the same scales of space-time. The principle of uncertainty in the sense stated before within the united system of $\left(H_{n \ell}, \mathcal{H}_{n \ell}\right)$ and $\left(H_{m k}, \mathcal{H}_{m k}\right)$ holds only at this stage. Between the two independent systems $\left(H_{n \ell}, \mathcal{H}_{n \ell}\right)$ and $\left(H_{m k}, \mathcal{H}_{m k}\right)$, the physics seems to be following classical mechanics for the observer, and the pair potentials $V_{\alpha}\left(x_{\alpha}\right)$ effective between these two systems operate as the classical

ones as in electromagnetism. One system $\left(H_{n \ell}, \mathcal{H}_{n \ell}\right)$ of these systems plays the role of the observer system, and another $\left(H_{m k}, \mathcal{H}_{m k}\right)$ is an observed system. In this case the coordinate system is $\left(x^{\lambda}\right)_{\lambda=0}^{3}=(t, x)=\left(t_{\left(H_{n \ell}, \mathcal{H}_{n \ell}\right)}, x_{\left(H_{n \ell}, \mathcal{H}_{n \ell}\right)}\right)$. By axioms 4 and 5 the 
motion of the centre of mass of the observed system relative to the observer system, when we neglect the effects other than the gravitational force, is thus expressed as in the theory of general relativity by the equation of motion

$$
\frac{d^{2} x^{\lambda}}{d \tau^{2}}+\sum_{\mu, \nu=0}^{3} \Gamma_{\mu \nu}^{\lambda} \frac{d x^{\mu}}{d \tau} \frac{d x^{\nu}}{d \tau}=0
$$

where $\tau=t_{m k}=t_{\left(H_{m k}, \mathcal{H}_{m k}\right)}$ is the proper time for the centre of mass of the system $\left(H_{m k}, \mathcal{H}_{m k}\right)$ and $\Gamma_{\mu \nu}^{\lambda}=1 / 2 \sum_{\alpha=0}^{3} g^{\lambda \alpha}\left(\partial g_{\alpha \mu} / \partial x^{\nu}+\partial g_{\nu \alpha} / \partial x^{\mu}-\partial g_{\mu \nu} / \partial x^{\alpha}\right)$ is the Christoffel's symbol.

We have to note that the above process of $\langle\langle$ observations $\rangle\rangle$ is concerned only with the classical-mechanical phenomena between the centres of mass of local systems. As we have stated, the observation is restricted only to the classical relative motions of centres of mass of local systems. However, the observer's system can also 〈〈see $\rangle$ the unobservable quantum-mechanical effects inside the observed system $\left(H_{m k}, \mathcal{H}_{m k}\right)$. Reversely to the classical-mechanical observations, the observer sees that the physics within the observed system is described by the Hamiltonian $H_{m k}$ in a quantum-mechanical way. The observer can $\langle\langle$ see $\rangle\rangle$ the clock and motions in the observed system through the classical observations using, e.g., light as in the astronomical observation of electromagnetic radiations from stars. The observer deduces from these classical observations, with some relativistic corrections of the observed values as will be discussed below, that the quantum-mechanical laws hold within the system $\left(H_{m k}, \mathcal{H}_{m k}\right)$, as far as the time of the system is defined as the quantum-mechanical proper one of the system $\left(H_{m k}, \mathcal{H}_{m k}\right)$ as in definition 3.

The results obtained in the usual relativistic quantum theories could be explained in our context as follows: The quantum phenomena occurring in a local system follow nonrelativistic quantum mechanics, but the observed values of quantum-mechanical quantities should be corrected according to the classical relativity so that the corrected values equal the values predicted by the (non-relativistic) quantum-mechanics. The relativistic effects arise only related with the observation of the classical quantities of the local system. The so-called propagation of fields or forces is interpreted in our theory also as the classical phenomena which appear through the observation process. What the observer measures concerning the observed system $\left(H_{m k}, \mathcal{H}_{m k}\right)$ is the classical-physics values of the subsystems or the portions of $\left(H_{m k}, \mathcal{H}_{m k}\right)$, e.g., the positions, momenta, etc., of the subsystem's centres of mass, measured according to the observer's local time. From these classical quantities, the observer deduces through the classical relativistic corrections that quantum mechanics is working in the observed system $\left(H_{m k}, \mathcal{H}_{m k}\right)$.

If we see this procedure in reverse order, as in the last but two paragraph of sect. 5, beginning with quantum mechanics, i.e. with the Schrödinger propagator $\exp \left[-i t_{m k} H_{m k}\right]$, given the system $\left(H_{m k}, \mathcal{H}_{m k}\right)$ with coordinates $\left(t_{m k}, x_{m k}\right)=\left(t_{\left(H_{m k}, \mathcal{H}_{m k}\right)}, x_{\left(H_{m k}, \mathcal{H}_{m k}\right)}\right)$, it is described more precisely as follows: The quantum-mechanical velocities of the particles in the system $\left(H_{m k}, \mathcal{H}_{m k}\right)$ are given by the quotients $q_{b}=x_{b} / t_{m k}$, asymptotically as $t_{m k} \rightarrow \infty$, of the position vectors $x_{b}$ of the particles and the local time $t_{m k}$. This is the case, since we have assumed that the coordinates $\left(t_{m k}, x_{m k}\right)$, in particular the local time $t_{m k}$, are given through the propagator or clock $\exp \left[-i t_{m k} H_{m k}\right]$ and axiom 2. Our fundamental assumption here on the observation is as follows: 
Axiom 6. The momenta $p_{j}=m_{j} x_{j} / t_{m k}$ of the particles $j$ with mass $m_{j}$ in the observed local system $\left(H_{m k}, \mathcal{H}_{m k}\right)$ with coordinate system $\left(t_{m k}, x_{m k}\right)$, given as above, are observed, by the observer system $\left(H_{n \ell}, \mathcal{H}_{n \ell}\right)$ with coordinate system $\left(t_{n \ell}, x_{n \ell}\right)$, as $p_{j}^{\prime}=m_{j} x_{j}^{\prime} / t_{n \ell}$, where $x_{j}^{\prime}$ is obtained from $x_{j}$ by the relativistic transformation of coordinates: $\left(t_{m k}, x_{m k}\right)$ to $\left(t_{n \ell}, x_{n \ell}\right)$ as in axiom 4 . The same is true for the observation of the energies of the particles: the energies of the particles in the observed local system are observed by the observer as the ones transformed in accordance with the relativity.

Namely, it is assumed that the quantum-mechanical momenta $p_{j}=m_{j} x_{j} / t_{m k}$ of the particles within the system $\left(H_{m k}, \mathcal{H}_{m k}\right)$ are observed in actual experiments by the observer system $\left(H_{n \ell}, \mathcal{H}_{n \ell}\right)$ with coordinate system $\left(t_{n \ell}, x_{n \ell}\right)$, as the classical quantities $p_{j}^{\prime}=m_{j} x_{j}^{\prime} / t_{n \ell}$ whose values are calculated or predicted by correcting the quantummechanical values $p_{j}$ with taking the relativistic effects of observation into account. A similar assumption is made for the energies of the particles.

In this sense, axiom 6 should be called a principle of deduction of the experimentally observed values from the non-relativistic quantum mechanics through certain relativistic corrections, rather than be called a deduction rule of the internal quantum-mechanical motions from the classical observations of the sublocal systems. We adopted the latter expression in sect. 2 and 6 for the sake of simplicity of expression.

We should note that axiom 6 is consistent with our axioms 1-5 in the following sense: axiom 6 is concerned only with the quantum mechanics within the local system $\left(H_{m k}, \mathcal{H}_{m k}\right)$, so that it gives the rules to transform the quantum-mechanical values, e.g. $p_{j}$, of the system $\left(H_{m k}, \mathcal{H}_{m k}\right)$ to the values, e.g. $p_{j}^{\prime}$, observed experimentally by the observer. It is therefore not related with any physics laws of the particles within the system $\left(H_{m k}, \mathcal{H}_{m k}\right)$, unless the transformed values $\left(e . g . p_{j}^{\prime}\right)$ are compared with the actual experimental values. In this sense, axiom 6 is concerned only with how the nature looks at the observer. Together with axioms 1-5, it gives the prediction of the physical values observed in actual experiments, and is checked solely through the experimental data.

In this sense, axiom 6 together with axioms 1-5 gives our prediction for the relativistic observations of the quantum-mechanical local systems. For the illustration, let us take an example of the calculation of the differential cross-section $d \sigma / d \Omega$ for the scattering phenomenon of an electron by a Coulomb potential $Z e^{2} / r$, where $r=|x|$ and $x$ is the position vector of the electron relative to the scatterer. We assume that the scatterer has a very large mass compared to the electron and that $Z / 137$ is small. Then, as usual, quantum mechanics gives, in a Born approximation,

$$
\frac{d \sigma}{d \Omega}=\frac{Z^{2} e^{4}}{16 E^{2} \sin ^{4}(\theta / 2)},
$$

where $\theta$ is the scattering angle, and $E$ is the total energy of the system consisting of the electron and the scatterer. Thus, since the electron is far away from the scatterer after the scattering, we may assume that the energy $E$ is equal to the kinetic energy of the electron and the scatterer. We assume that the observer is stationary relative to this local system of the electron and the scatterer. This means that the observer is approximately stationary relative to the scatterer, since we assumed the mass of the scatterer much larger than that of the electron. From the observer, which is stationary relative to the 
scatterer, the energy $E$ is equal to the kinetic energy of the electron and is observed as a classical quantity by axiom 6 . Therefore, its actual observed value equals $p^{0}-p_{0}^{0}$ by special relativity. Here $p_{0}^{0}$ is the rest energy of the electron, and $p^{0}$ is the relativistic energy of the electron given as follows. Let $v$ be the absolute value of the coordinate velocity of the electron: $v=|d x / d t|$, where $t$ is the observer's local time. Then, by the theory of special relativity, $p^{0}$ is expressed as follows:

$$
p^{0}=\frac{1}{\sqrt{1-v^{2}}} p_{0}^{0}
$$

Here we adopted a unit system such that the speed of light $c=1(>v \geq 0)$. Thus $p_{0}^{0}$ equals the rest mass $m_{0}$ of the electron: $p_{0}^{0}=m_{0}$. We can then compute

$$
E=p^{0}-p_{0}^{0}=\frac{1-\sqrt{1-v^{2}}}{\sqrt{1-v^{2}}} p_{0}^{0} \approx \frac{m_{0} v^{2} / 2}{\sqrt{1-v^{2}}} \approx \frac{m_{0} v^{2}}{2}\left(1+v^{2} / 2\right) \approx \frac{m_{0} v^{2}}{2} .
$$

Thus, taking the relativistic effects of the observation into account, we have the differential cross-section given by

$$
\frac{d \sigma}{d \Omega} \approx \frac{Z^{2} e^{4}}{4 m_{0}^{2} v^{4} \sin ^{4}(\theta / 2)}\left(1-v^{2}\right)
$$

This coincides with the usual relativistic prediction of the Klein-Gordon equation obtained by a Born approximation, if the observer is assumed to be stationary relative to the scatterer.

The effect of the spin of the electron can also be included by introducing the spin-orbit interactions (see Mott-Massey [18], chapt. X). In this treatment, the wave function of the electron is regarded as a two-dimensional vector-valued function as usual. The result corresponding to (14) is the same as the one obtained through Dirac theory as far as the spin correction is concerned (Mott-Massey [18], chapt. X, sect. 3):

$$
\frac{d \sigma}{d \Omega}=\frac{Z^{2} e^{4}}{16 E^{2} \sin ^{4}(\theta / 2)}\left(1-\frac{2 E}{m_{0}} \sin ^{2}(\theta / 2)\right) .
$$

Inserting the relativistic correction (16), we get

$$
\frac{d \sigma}{d \Omega} \approx \frac{Z^{2} e^{4}}{4 m_{0}^{2} v^{4} \sin ^{4}(\theta / 2)}\left(1-v^{2} \sin ^{2}(\theta / 2)\right)\left(1-v^{2}\right)
$$

This is exactly the relativistic Dirac prediction in a Born approximation. We can proceed to finer Born approximations as well, as in Mott-Massey [18], chapt. IX, sect. 4.5, and can recover the relativistic prediction of Dirac equation.

In sum, quantum mechanics has the intrinsic nature, and relativity is concerned with how nature looks at the observer. The observed relativistic quantum phenomena are explained as the consequences of the relativistic effects of the observation of the nonrelativistic quantum systems. 


\section{0. - Concluding discussions.}

The times are defined only for local systems $\left(H_{n \ell}, \mathcal{H}_{n \ell}\right)$. The total universe $\phi$ has no time associated. The local times arise through the affections from other particles outside the local systems (definitions 1-3). The uncertainty principle holds only within these local systems as the uncertainty of the local times. Quantum mechanics is confined within each local system in this sense. The quantum-mechanical phenomena between two local systems appear only when they are combined as a single local system. In the local system, the interaction and forces propagate with infinite velocity or, in other words, they are unobservable.

Each local system can be the observer of other systems. In this situation, the local systems are mutually independent in the sense that the associated quantum-mechanical local times are not correlated in general. Therefore, there are no reasons to exclude classical mechanics in describing the observable relative behaviour of the observed systems with respect to the observer. Thus, the gravitational potentials can be introduced in accordance with the theory of general relativity. These potentials determine the global space-time structure around the observer system. Inside the observer system the spacetime is Euclidean. The observer itself cannot detect the gravitational correlation or the space-time structure inside its own system, and the interactions and forces cannot be detected inside it. On the contrary, between the local systems, the observer can detect only the classical-mechanical effects. Namely, the gravitational forces appear and the quantum-mechanical potentials $V_{\alpha}\left(x_{\alpha}\right)$ effective outside the local systems operate as the classical ones between the local systems. Nevertheless, through the media (e.g., light in classical sense) which connect the observer and the observed systems and obey the classical physics, the observer sees, through some relativistic corrections of the observed classical values, that the physics laws inside the other local systems follow quantum mechanics.

These facts are all the consequences of the introduction of local times which are proper to each local system. The time is neither a given thing nor a common one to the total universe. On the contrary, there can be defined no global time. More strongly the total universe is a (stationary) bound state of the total Hamiltonian $H$ of infinite degrees of freedom. The times arise only when the observers restrict their attention to its subsystems as approximations of the total Hamiltonian $H$. The universe itself is correlated within it as a bound state of $H$. The observer always separates a subsystem from it, so to speak, artificially, and the (steady) motion and time appear. Inside the subsystem this local time explains the quantum effects, and outside the subsystem it explains the gravitation and the classical mechanics. The relativistic quantum phenomena are explained as the relativistic effects of the observation of the non-relativistic quantum systems. All these physical phenomena occur by this artificial separation of the universe. The universe itself does not $\langle\langle$ change $\rangle\rangle$ : It is a stationary bound state.

\section{1. - Some conjectures.}

As a conclusion, we state some conjectures which would likely hold in our theory.

1) The universe $\phi$ would be confined in a local region of the infinite-dimensional configuration space $R^{\infty}$ in some sense as an eigenvector of the total Hamiltonian $H$ : This 
is an analogy with the corresponding fact for the finite-dimensional Hamiltonian $H_{n \ell}$. The eigenvectors for this Hamiltonian are local in the sense that they are in $L^{2}\left(R_{x^{(\ell)}}^{3 n}\right)$. Or, more strongly, it is known that they decay exponentially with respect to $\left|x^{(\ell)}\right|$ if the associated eigenvalues are not the thresholds of $H_{n \ell}$. This conjecture means that the universe is a $\langle\langle$ closed $\rangle\rangle$ or $\langle\langle$ finite $\rangle\rangle$ one in quantum-mechanical sense. Therefore, this conjecture will eliminate the arguments on the so-called $\langle\langle$ dark matter $\rangle\rangle$, which is supposed to exist in the usual $\langle\langle$ big-bang $\rangle\rangle$ theory to make the universe almost stable, i.e. to make it have the density very close to the $\langle\langle$ critical density $\rangle\rangle$, therefore to let the universe have matters enough for stars and planets to exist. A similar explanation will work for explaining the stability of such local systems $\left(H_{n \ell}, \mathcal{H}_{n \ell}\right)$ as galaxies, clusters of galaxies, etc., if one regards their actual quantum-mechanical states $\phi_{n \ell} \in \mathcal{H}_{n \ell}$ as (approximate) bound states of the local Hamiltonian $H_{n \ell}$, namely as $\left\langle\langle\right.$ resonances $\rangle$ of $H_{n \ell}$ in some sense (cf. Jensen-Kato [19]). Then, the quantum-mechanical effect would explain their stability or $\langle\langle$ locality $\rangle\rangle$ as in the explanation of the locality of the total universe without introducing the dark matter.

2) It would be possible to accommodate bosons, fermions, photons (i.e. the quantum theory of radiation), and so on in our theory by using sub-Fock spaces $\mathcal{F}=\bigoplus_{n=0}^{\infty} \mathcal{H}^{n}$ of $\mathcal{U}=\bigoplus_{n=0}^{\infty}\left(\sum_{\ell=0}^{\infty} \mathcal{H}^{n}\right)$. As usual the spin of the particles can be introduced by taking the vector-valued representations $L^{2}\left(R^{3 n} ; \mathbf{C}^{k}\right)(k \geq 1)$ of $\mathcal{H}^{n}$ in axiom 2. ( $\mathbf{C}$ is the set of complex numbers.)

For the photons without interactions with matter, the Hamiltonians $H_{n \ell}$ in the sense of axiom 3 should be taken as a sum of those for harmonic oscillators. Since the corresponding total Hamiltonian $H_{p}=\bigoplus_{n=0}^{\infty} H_{n \ell}$ has a complete system of eigenvectors, one has $\left(I-P_{H_{p}}\right) \mathcal{F}=\{0\}$. Thus it has no associated proper clock. In this sense the light as a wave propagates with infinite speed within the system $\left(H_{p}, \mathcal{F}\right)$. It should be remarked that this statement has a different meaning than the statement that the light, as an electromagnetic interaction, propagates with infinite speed. As such a field, light interacts with other matters through vector potentials $A(x)$, which are quantum-mechanical electromagnetic fields interacting instantaneously with matters. On the contrary, as a wave, light propagates with the constant speed $c$ within general local systems. The reason is that the Hamiltonian of a general local system contains terms comprising the interactions between photons and other particles. As a consequence, that Hamiltonian does not have a complete system of eigenvectors, and the local time of the system is defined by definition 3. Then, with some additional arguments to chapt. III of von Neumann [20], one can show that the speed of the wave front of light within that local system is the constant $c$.

In the line of this treatment of the interactions between electromagnetic field and matters, it would be possible to explain the Lamb shift. It is probable that the explanation could be given even without appealing to the path integral techniques described, e.g., in Feynman-Hibbs [21]. For this possibility, we refer to the argument around the footnote 149) of [20], chapt. III, sect. 6, remarking that the argument there and the one in [21], chapt. 9, are essentially the same. As in [20], let $H$ be the total Hamiltonian of the system consisting of matter and radiation field (i.e. photons), let $I$ be the interaction between matter and photons, and let a sequence of complex numbers $a_{k M_{1} M_{2} \ldots}$ belong to the space of sequences $a_{k M_{1} M_{2} \ldots}$ with normalization condition that $\sum_{k, M_{1}, M_{2}, \ldots}\left|a_{k M_{1} M_{2} \ldots}\right|^{2}=1$ and let its component $a_{k M_{1} M_{2}} \ldots$ express the state where matter is in the state $k$ and the 


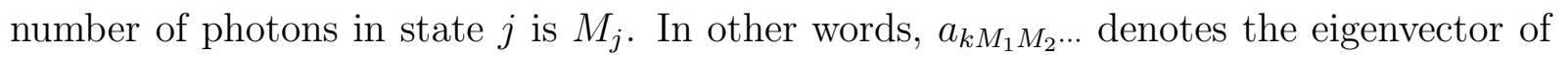
$H-I$ with eigenvalue $W_{k}+\sum_{j=1}^{\infty} h \rho_{j} \cdot M_{j}$, where $W_{k}$ is the eigenenergy of the matter state $k, \rho_{j}$ is the eigenfrequency of photons in state $j$, and $h$ is the Planck constant. Set $b_{k M_{1} M_{2} \ldots}(t)=\exp \{-i t H\} \exp \{i t(H-I)\} a_{k M_{1} M_{2} \ldots .}$ Consider the transition process where the matter part of the total system begins and ends in the same state $\bar{k}$. Integrating the differential equation

$$
\frac{1}{i} \frac{d}{d t} b(t)=\exp \{-i t H\}(-I) \exp \{i t H\} b(t), \quad b(0)=a_{\bar{k} \bar{M}_{1} \bar{M}_{2} \cdots}
$$

in some approximation, and estimating the solution, as in [20], chapt. III, sect. 6, we see that

$$
\sum_{\forall M_{j}}\left|b_{\bar{k} M_{1} M_{2} \ldots}(t)\right|^{2}=1-\sum_{k \neq \bar{k}, \forall M_{j}}\left|b_{k M_{1} M_{2} \ldots}(t)\right|^{2}
$$

with the second term on the right-hand side $\geq$ const. $|t|($ const. $\neq 0)$ in norm for small $|t|$.

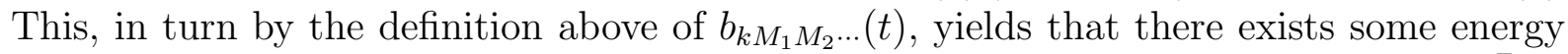
shift $\delta E \neq 0$ to the total energy of the system during the transition from the state $\bar{k}$ to the same state $\bar{k}$ of the matter part of the system. In this sense, it seems that the Lamb shift was already predicted at least implicitly around 1930. We expect that this kind of argument could be refined in rigorous sense to give the explanation of the Lamb shift with no divergence problem, in our context of abandonment of relativistic quantum field theory.

These conjectures stated here do not require us to introduce the notion of relativistic quantum field, because the system should be considered as a non-relativistic one. (See also Dirac [22], chapt. X other than [20], chapt. III.) The relativistic quantum phenomena could be explained as the relativistic effects of observations as discussed in the section on observation.

3) We adopt the standpoint that quantum mechanics is symmetric with respect to the reversal of (local) time: $t \rightarrow-t$. In this sense we stand upon the continuous 〈〈Schrödingerlike $\rangle$ picture concerning the measurement of the local systems by the observer's local system: As far as the local systems $\left(H_{n \ell}, \mathcal{H}_{n \ell}\right)$ consisting of finite number of particles are concerned, no bound state $\psi_{n \ell}$ of $H_{n \ell}$ in $\mathcal{H}_{n \ell}$ with eigenvalue $\mu$ can be detected if one observes the system in accord with the local time $t=t_{\left(H_{n \ell}, \mathcal{H}_{n \ell}\right)}$, because the state $\psi_{n \ell}$ does not change in its amplitude: $\left|e^{-i t H_{n \ell}} \psi_{n \ell}(x)\right|^{2}=\left|e^{-i t \mu} \psi_{n \ell}(x)\right|^{2}=\left|\psi_{n \ell}(x)\right|^{2}$. Therefore, it emits no light and information outside. Namely, only the scattering state which lies in the continuous spectral subspace $\mathcal{H}_{n \ell}^{c}\left(\subset \mathcal{H}_{n \ell}\right)$ of the local Hamiltonian $H_{n \ell}$ can be detected. We need not consider here the so-called reduction of the wave packets. The observer measures only, e.g., the light emitted from the scattering state in the observed system $\left(H_{n \ell}, \mathcal{H}_{n \ell}\right)$. In this sense the measurement does not include the irreversible procedure in our theory, and the (local) time is reversible. (We refer to von Neumann [20], chapt. III, VI for the arguments which support our standpoint including the explanation of emission of light in the line of conjecture (2).)

4) By the consistency of axioms 4-5 with axioms 1-3, the theory of (general) relativity implies Hubble's red-shift. Namely, the universe looks like it is expanding (or contracting) 
for any observer's local system. At the same time, axioms 1-3 and definitions 1-3 imply that the universe has no proper time associated and is stationary. This situation is no contradiction, because axioms 1-5 are consistent. The expansion appears only when the observer's local system observes the universe in accordance with classical mechanics. In other words, the universe looks like expanding only when the observer puts its concerns on the centres of mass of the stars, galaxies, etc., regarding them as other local systems. Only in this context it is required to argue on the beginning and the end of the universe. But an appearance of the universe as this comes from the viewpoint merely based on classical mechanics. When the observer looks at the inside of those local systems, he sees that quantum mechanics is working inside them as we have seen in sect. 9. Then he reasons from these observations and consideration that the universe itself is a quantummechanical one, and concludes that the consistent introduction of quantum mechanics into such a situation would remove the problem of the beginning and the end of the

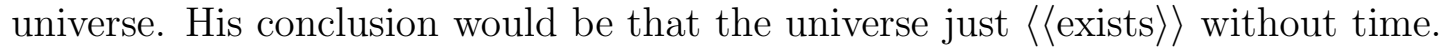

\section{References}

[1] A. Ashtekar, J. Stachel (eds.), Conceptual Problems of Quantum Gravity, Birkhäuser, Boston-Basel-Berlin, 1991.

[2] H.R. Brown, R. Harré (eds.), Philosophical Foundations of Quantum Field Theory, Clarendon Press, Oxford, 1990.

[3] J. Glimm, A. Jaffe Quantum Physics, A Functional Integration Point of View, SpringerVerlag, 2nd edn., New York-Berlin-Heidelberg-London-Paris-Tokyo, 1987.

[4] M. Redhead, Incompleteness, Nonlocality, and Realism, A Prolegomenon to the Philosophy of Quantum Mechanics, Clarendon Press, Oxford, 1990.

[5] E. Prugovečki, Quantum Geometry, A Framework for Quantum General Relativity, Kluwer Academic Publishers, Dordrecht-Boston-London, 1992.

[6] R. Abraham, J.E. Marsden, Foundations of Mechanics, The Benjamin/Cummings Publishing Company, 2nd edn., London-Amsterdam-Don Mills, Ontario-Sydney-Tokyo, 1978.

[7] H. Kitada, Asymptotic completeness of $N$-body wave operators I. Short-range quantum systems, Rev. Math. Phys. 3, 1991, 101-124.

[8] V. Enss, Introduction to asymptotic observables for multiparticle quantum scattering, in "Schrödinger Operators, Aarhus 1985," ed. E. Balslev, Lect. Note in Math. 1218, SpringerVerlag, 1986, pp.61-92.

[9] H. Kitada, Asymptotic completeness of N-body wave operators II. A new proof for the shortrange case and the asymptotic clustering for long-range systems, in "Functional Analysis and Related Topics, 1991," ed. H. Komatsu, Lect. Note in Math. 1540, Springer-Verlag, 1993, pp.149-189.

[10] S.W. Hawking, G.F.R. Ellis, The Large Scale Structure of Space-Time, Cambridge University Press, Cambridge-New York-Port Chester-Melbourne-Sydney, 1973. 
[11] J. Dereziński, Asymptotic completeness of long-range N-body quantum systems, preprint (Centre de Mathématiques, Ecole Polytechnique, U.R.A. 169 du C.N.R.S.), 1992.

[12] A. Einstein, Die Grundlage der allgemeinen Relativitätstheorie, Ann. der Phys. (Leibzig), Ser. 4, 49, 1916, 769-822.

[13] M. Friedman, Foundations of Space-Time Theories, Relativistic Physics and Philosophy of Science, Princeton University Press, Princeton, 1983.

[14] A. Einstein, B. Podolsky, N. Rosen, Can quantum-mechanical description of physical reality be considered complete?, Phys. Rev. 47, 1935, 777-780.

[15] M. Jammer, The Philosophy of Quantum Mechanics, The interpretations of Quantum Mechanics in Historical Perspective, John Wiley \& Sons, Inc., New York, 1974.

[16] F. Selleri, Die Debatte um die Quantentheorie, Frieder. Vieweg \& Sohn Verlagsgesellschaft mbH, Braunschweig, 1983.

[17] W. Schommers (ed.), Quantum Theory and Pictures of Reality, Foundations, Interpretations, and New Aspects, (with contributions by B. d'Espagnat, P. Eberhard, W. Schommers, F. Selleri), Springer-Verlag, Berlin-Heidelberg-New York-London-Paris-Tokyo-Hong Kong, 1989.

[18] N.F. Mott, H.S.W. Massey, The Theory of Atomic Collisions, Clarendon Press, 3rd edn., Oxford, 1965.

[19] A. Jensen, T. Kato, Spectral properties of Schrödinger operators and time-decay of the wave functions, Duke Math. J. 46, 1979, 583-611.

[20] J. von Neumann, Die Mathematische Grundlagen der Quantenmechanik, Springer-Verlag, Berlin, 1932.

[21] R.P. Feynman, A.R. Hibbs, Quantum Mechanics and Path Integrals, McGraw-Hill Book Company, New York-St. Louis-San Francisco-Toronto-London-Sydney, 1965.

[22] P.A.M. Dirac, The Principles of Quantum Mechanics, Clarendon Press, 4th edn., Oxford, 1958. 